\title{
FUNCIONALIZAÇÃO DE NANOTUBOS DE CARBONO
}

\author{
Antônio Gomes de Souza Filho* \\ Departamento de Física, Universidade Federal do Ceará, CP 6030, 60455-900 Fortaleza - CE, Brasil \\ Solange Binotto Fagan \\ Área de Ciências Naturais e Tecnológicas, Centro Universitário Franciscano, 97010-032 Santa Maria - RS, Brasil
}

Recebido em 28/7/06; aceito em 18/1/07; publicado na web em 25/9/07

\begin{abstract}
FUNCTIONALIZATION OF CARBON NANOTUBES. Carbon nanotubes are very stable systems having considerable chemical inertness due to the strong covalent bonds of the carbon atoms on the nanotube surface. Many applications of carbon nanotubes require their chemical modification in order to tune/control their physico-chemical properties. One way of achieving this control is carrying out functionalization processes where atoms and molecules interact (covalent or non-covalent) with the nanotubes. We review some of the progress that has been made in chemical functionalization of carbon nanotubes. Emphasis is given to chemical strategies, the most used techniques, and applications.
\end{abstract}

Keywords: carbon nanotubes; functionalization; nanomaterials.

\section{INTRODUÇÃO}

Os nanomateriais, em geral, são muito importantes porque se diferenciam de forma dramática dos seus precursores "bulk". As propriedades destes materiais são determinadas pelo tamanho e pela morfologia, originando uma fascinante sintonia em suas propriedades físico-químicas. Talvez os exemplos mais claros e ilustrativos desses fenômenos possam ser tomados da ciência do carbono após a descoberta dos fulerenos, em $1985^{1}$, e dos nanotubos de carbono, em $1991^{2}$. Os nanotubos de carbono, assunto dessa revisão, são sistemas modelo para a nanociência e a nanotecnologia. Essas novas estruturas de carbono são bastante versáteis para se integrarem a diferentes áreas do conhecimento e são capazes de promover uma inter/multidisciplinaridade muito forte. Hoje, as pesquisas em nanotubos de carbono cruzam as fronteiras da física, da química, das ciências dos materiais, da biologia e desenvolvem-se rapidamente no campo da farmacologia.

Os nanotubos de carbono, quanto ao número de camadas, podem ser classificados em duas formas: nanotubos multicamadas ("multi-wall carbon nanotubes - MWNTs") e camada simples ("single-wall carbon nanotubes - SWNTs"). Um tipo especial de MWNT é o nanotubo de parede dupla ("double-wall carbon nanotubes - DWNTs"). Uma ou outra forma de nanotubos apresenta-se mais apropriada dependendo da aplicação desejada. Os MWNTs foram observados pela primeira vez por Iijima², em 1991. Dois anos depois, em contribuições independentes, Iijima e colaboradores $^{3}$ no Japão e Bethune e colaboradores ${ }^{4}$ nos EUA publicaram simultaneamente a síntese dos SWNTs. Recentemente debate-se a quem devem ser atribuídos os créditos da descoberta dos nanotubos de carbono ${ }^{5}$. Entretanto, não há dúvidas que a área de pesquisa em nanotubos consolida-se após o trabalho de Iijima $^{2}$. A Figura 1 mostra imagens de microscopia eletrônica de transmissão (TEM) de MWNTs, DWNTs e SWNTs. O avanço das pesquisas em nanotubos de carbono foi claramente impulsionado pelo desenvolvimento dos processos de síntese, levando à produção de feixes de nanotubos do tipo SWNT com boa qualidade. Esse avanço foi realizado pelo grupo do Prof. Smalley na Rice University ${ }^{6} \mathrm{e}$

*e-mail: agsf@fisica.ufc.br tornou possível a execução de estudos de microscopia ${ }^{7,8}$ e de espectroscopia ${ }^{9}$ que permitiram comprovar as principais propriedades físicas dos nanotubos, previstas no início da década de $90^{10,11}$. Hoje, diversos métodos de preparação de nanotubos de carbono foram desenvolvidos e podem ser produzidos ${ }^{12}$ nanotubos isolados sobre substratos, suspensos em pilares e dispostos em arquiteturas complexas.

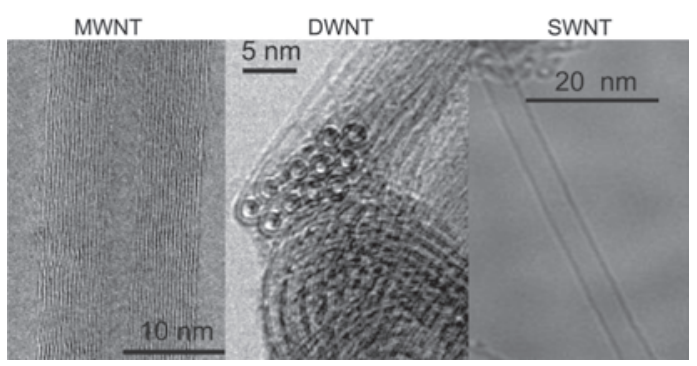

Figura 1. Imagens de microscopia eletrônica de transmissão de nanotubos MWNT, DWNT e SWNT

Os nanotubos de carbono apresentam propriedades eletrônicas, óticas e mecânicas muito interessantes ${ }^{13}$. Desta forma, esses materiais têm sido usados na confecção de diferentes tipos de dispositivos, como emissores de elétrons para mostradores, sensores de gases e sensores biológicos, pontas para microscópio de força atômica (AFM) e, quando combinados a outros materiais, como polímeros e fibras, servem como elementos de reforço formando compósitos com excelentes propriedades mecânicas ${ }^{14}$.

A funcionalização de nanotubos de carbono através de suas paredes, pontas ou por encapsulamento (os tubos de pontas abertas possuem capilaridade) tem sido vista como uma forma de explorar o potencial dos nanotubos de carbono na nanotecnologia. Os nanotubos funcionalizados podem ter propriedades eletrônicas e mecânicas que são substancialmente diferentes dos nanotubos não funcionalizados e este fenômeno é explorado para uso em sensores, dispositivos eletrônicos e eletro-mecânicos em escala nanométrica devido a sua grande resistência e flexibilidade mecânica. Essas estruturas quimicamente modificadas podem ser usadas de forma a facilitar a interação dos nanotubos com moléculas orgânicas e 
biológicas ${ }^{15}$, com outros grupos químicos como fármacos ou moléculas tóxicas ${ }^{16-19} \mathrm{e}$, até mesmo, com vírus e bactérias ${ }^{20}$, tornadoos sensores capazes de detectar pequenos traços da espécie alvo e com alta seletividade. Certamente, o desenvolvimento de sensores utilizando nanotubos de carbono funcionalizados como bloco de construção é uma das áreas mais promissoras para uso desses materiais em nanotecnologia. O desafio é encontrar rotas quimicamente seguras, limpas e factíveis para alterar os nanotubos de carbono que em seu estado natural apresentam reatividade química muito baixa ${ }^{21}$.

\section{CONCEITOS E PROPRIEDADES DOS NANOTUBOS DE CARBONO}

Será apresentado um resumo das intrigantes propriedades físicas dos nanotubos de carbono, iniciando com as propriedades estruturais e seguindo com as propriedades eletrônicas, vibracionais e óticas.

\section{Estrutura atômica}

Um nanotubo de carbono é conceitualmente construído como sendo formado a partir de uma folha de grafite (grafeno) enrolada em forma cilíndrica, com "um átomo de espessura", como ilustrado na Figura $2 \mathrm{a}^{22}$. Apesar de muitos progressos terem sido realizados no aprimoramento dos diferentes métodos de síntese dos nanotubos, o entendimento do processo de crescimento ainda não atingiu um estágio onde seja possível controlar a estrutura do nanotubo. É importante notar que a maneira pela qual a folha de grafeno é enrolada determina a estrutura dos nanotubos e suas propriedades físicas. Os dois parâmetros estruturais relevantes dos nanotubos são: diâmetro $\left(d_{t}\right)$ e ângulo quiral $(\theta)$ (também chamado de quiralidade ou helicidade). Usando a estrutura de uma rede hexagonal bidimensional (Figura 2b), pode-se descrever, em primeira aproximação, a estrutura dos nanotubos.

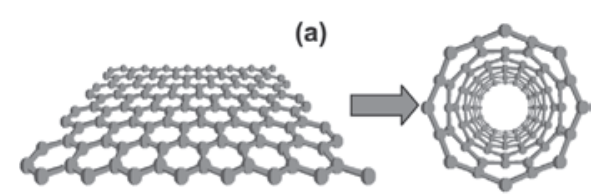

(b)

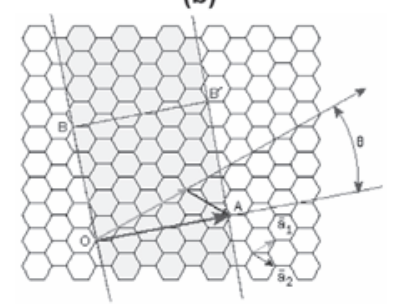

(c)

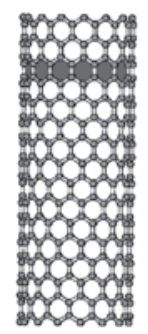

$(15,0)$

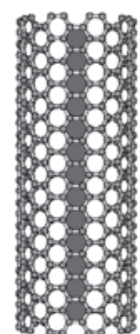

$(9,9)$

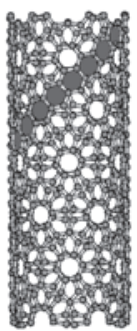

$(12,6)$
Figura 2. (a) Diagrama ilustrando como um nanotubo de carbono é formado a partir da folha de grafite; (b) a definição do vetor quiral, para o caso $\mathrm{n}=4$ e $\mathrm{m}=2$. (c) Nanotubos com $\theta=0^{\circ}(15,0) \theta=30^{\circ}(9,9)$ e $\theta=19.0^{\circ}(12,6)$
Define-se o vetor quiral $\mathbf{C}_{\mathrm{h}}$ como sendo uma combinação dos vetores de base $\left(\mathbf{a}_{1}\right.$ e $\left.\mathbf{a}_{2}\right)$ da rede do grafeno. $\mathrm{O}$ vetor quiral $\mathbf{C}_{\mathrm{h}}$ conecta dois pontos cristalograficamente equivalentes da rede hexagonal sendo definido como

$\mathbf{C}_{\mathrm{h}}=\mathrm{n} \mathbf{a}_{1}+\mathrm{m} \mathbf{a}_{2} \equiv(\mathrm{n}, \mathrm{m})$

onde, $n$ e $m$ são inteiros. Os vetores $\mathbf{a}_{1}$ e $\mathbf{a}_{2}$ possuem módulos iguais sendo

$\left|\mathbf{a}_{1}\right|=\left|\mathbf{a}_{2}\right|=3^{1 / 2} \mathbf{a}_{\mathrm{C}-\mathrm{C}=} 3^{1 / 2} 1,42=2,46 \AA$

onde $a_{C-C}$ é a distância da ligação carbono-carbono na folha de grafite. Desprezando os efeitos de curvatura no comprimento das ligações $a_{C-C}$, o diâmetro do nanotubo pode ser determinado a partir do módulo do vetor quiral $\mathbf{C}_{\mathrm{h}}$. $\mathrm{O}$ comprimento da circunferência do nanotubo é $\left|\mathbf{C}_{\mathrm{h}}\right|=\pi \mathrm{d}_{\mathrm{t}}$. O módulo de $\mathbf{C}_{\mathrm{h}}$ é facilmente determinado em termos de $a_{C-C}$ e dos índices $n$ e $m$ :

$\mathrm{d}_{\mathrm{t}}=\left|\mathbf{C}_{\mathrm{h}}\right| / \pi=\mathrm{a}\left(n^{2}+m^{2}+n m\right)^{1 / 2} / \pi$

O cálculo do diâmetro realizado usando-se a Equação 3 negligencia os efeitos da curvatura no comprimento de ligações C-C e é válido para nanotubos com diâmetros maiores que $1.0 \mathrm{~nm}$. Para nanotubos com grande curvatura (pequeno diâmetro) o cálculo de $d_{t}$ usando a Equação 3 não é mais uma boa aproximação.

O ângulo quiral ( $\theta$ ) é também obtido a partir dos índices $n$ e $m$ e é dado por

$\theta=\cos ^{-1}\left(\frac{n+m / 2}{\sqrt{n^{2}+n m+m^{2}}}\right)$

Os nanotubos com $\theta=0^{\circ}$ e $30^{\circ}$ são aquirais e denominados de "zig-zag" $(n, 0)$ e "armchair" $(n, n)$, respectivamente. Os nanotubos $\operatorname{com} 0^{\circ}<\theta<30^{\circ}$ são denominados de quirais. Na Figura 2c são mostrados os nanotubos com quiralidades $\theta=0^{\circ}$ [tubo $\left.(15,0)\right], \theta=30^{\circ}$ [tubo $(9,9)]$, e $\theta=19^{\circ}$ [tubo $\left.(12,6)\right]$. Uma análise completa das propriedades de simetria dos nanotubos usando teoria de grupos é apresentada nas refs. 23 e 24 .

\section{Estrutura eletrônica}

Existem diferentes métodos para se calcular as propriedades eletrônicas dos nanotubos de carbono. O método mais simples é determinar as propriedades dos nanotubos considerando-se as propriedades do grafeno. O grafeno possui um "gap" (separação entre as bandas de valência e condução) de energia nulo nos pontos $\mathrm{K}$ da zona de Brillouin, ou seja, a banda de valência toca a banda de condução nestes pontos ${ }^{22}$. Já para os nanotubos, os estados eletrônicos permitidos, comparados com o grafeno, são restritos, pois ao longo da circunferência do nanotubo existe uma quantização dos comprimentos de onda associados à função de onda dos elétrons. Somente são permitidos comprimentos de onda com uma relação de número inteiro com o comprimento da circunferência do nanotubo. Como os nanotubos são bastante longos (comprimento micrométrico) os vetores de onda ao longo do eixo são contínuos. Em geral, o caráter metálico ou semicondutor do nanotubo é determinado pelos índices $n$ e $m$. Devido às propriedades de simetria do grafeno $^{22,24}$ tem-se a seguinte classificação: se $n-m$ for múltiplo de 3 , o nanotubo é semicondutor de "gap" quase nulo e, se $n-m$ não for múltiplo de 3 o nanotubo é semicondutor. No caso especial onde $n=m$ o nanotubo é metálico. A Figura 3 mostra a densidade de estados eletrônicos de um nanotubo semicondutor (Figura 3a) e 
de um nanotubo metálico (Figura 3b). Devido à quantização dos estados eletrônicos ao longo da circunferência do nanotubo, a densidade de estados apresenta picos, deixando o sistema 1D com características quase moleculares. Os picos na banda de valência $\left(E_{1}(v)\right.$, $\left.\mathrm{E}_{2}(\mathrm{v}), \ldots\right)$ e de condução $\left(\mathrm{E}_{1}(\mathrm{c}), \mathrm{E}_{2}(\mathrm{c}), \ldots\right)$ são chamados de singularidades de van Hove. Para os nanotubos semicondutores, os estados preenchidos (estados de valência $\pi$ ) estão localizados abaixo do nível de Fermi e existe uma lacuna de energia ("gap") entre o primeiro estado preenchido da banda de valência e o primeiro estado vazio (estados de valência $\pi *$ ) da banda de condução. Para os nanotubos metálicos, o nível de Fermi é ocupado e não existe lacuna de estados entre os níveis vazios e preenchidos.

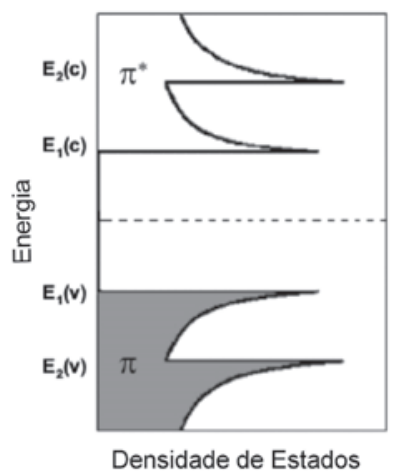

(a)

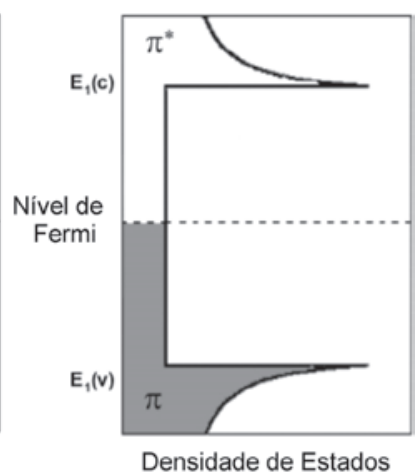

(b)
Figura 3. Densidade de estados eletrônicos para SWNTs semicondutores (a) e metálicos (b). Os estados preenchidos (orbitais $\pi$ ) estão localizados abaixo do nível de Fermi e os estados vazios (orbitais $\pi^{*}$ ) acima do nível de Fermi

\section{Propriedades vibracionais e óticas}

Devido ao confinamento quântico nos nanotubos as propriedades óticas e vibracionais são pronunciadas e unicamente determinadas pela estrutura atômica. Uma das técnicas mais utilizadas no estudo de nanotubos de carbono e que permitiu um grande avanço no entendimento das propriedades eletrônicas é a espectroscopia Raman ressonante. A versatilidade dessa técnica permite o estudo dos mais variados tipos de amostras de nanotubos de carbono (feixes de SWNT e MWNT, nanotubos em solução, nanotubos isolados, dispersos em compósitos etc ...). O espectro Raman dos nanotubos é observado quando a energia do laser usado na excitação dos espectros é muito próxima à energia das transições eletrônicas entre as singularidades de van Hove na banda de valência e na banda de condução, ou seja, $E_{\text {laser }} \sim E_{i i} \sim E_{i}(c)-E_{i}(v)$ onde $i=1,2,3$, ... . As transições $E_{i i}$ são permitidas para luz polarizada ao longo do eixo do tubo e as transições cruzadas do tipo $\mathrm{E}_{12}$ são permitidas para luz polarizada perpendicularmente ao eixo do nanotubo ${ }^{24}$. No entanto, neste último caso a intensidade é fortemente reduzida devido ao fato dos nanotubos apresentarem o efeito antena, ou seja, os fenômenos relacionados com a absorção de luz são predominantemente associados com a componente do campo elétrico paralelo ao eixo do nanotubo.

O volume de informação obtido com a espectroscopia Raman é considerável e é fundamental para o entendimento das propriedades eletrônicas e óticas dos nanotubos, de maneira relativamente simples. Para uma revisão detalhada sobre este assunto ver refs. 23 e 25. O espectro Raman é caracterizado por vários picos, sendo que associado a cada um deles existe uma grande riqueza de fenômenos agregados à estrutura eletrônica e vibracional do nanotubo. Os modos vibracionais mais estudados são ${ }^{23}$ : (a) modo radial de respiração (ocorre na região de baixo número de onda). O número de onda está relacionado com o diâmetro do nanotubo e $v_{\mathrm{RBM}}=\mathrm{A} / \mathrm{d}_{\mathrm{t}}+\mathrm{B}$, onde $\mathrm{A}$ e $\mathrm{B}$ variam dependendo do tipo de amostra; (b) a banda G, que ocorre entre 1400 e $1650 \mathrm{~cm}^{-1}$, fornece informações sobre o caráter metálico ou semicondutor do nanotubo ${ }^{26}$; (c) a banda D é o modo proveniente da desordem estrutural e só aparece devido à quebra de simetria translacional do nanotubo, a qual pode ser originada pela presença de um defeito ou de uma ligação com alguma molécula ${ }^{23,27}$.

$\mathrm{O}$ deslocamento em número de onda da banda $\mathrm{G}$ e a intensidade da banda $\mathrm{D}$ fornecem informações importantes sobre as mudanças na estrutura eletrônica dos nanotubos, quando interagem com espécies químicas. As frequiências desses modos são também bastante sensíveis ao processo de transferência de carga entre os nanotubos e espécies químicas ligadas aos nanotubos ou intercaladas entre os tubos.

As técnicas de absorção e emissão ótica também foram muito importantes no entendimento das propriedades dos nanotubos ${ }^{13} \mathrm{e}$ foram fundamentais para o entendimento das propriedades eletrônicas dos nanotubos em feixes. Os picos observados no espectro de absorção ótica correspondem às transições entre cada par de singularidades mostrado nos gráficos da densidade de estados na Figura 3. As energias observadas no espectro de absorção são: $E_{11}=E_{1}(c)-E_{1}(\mathrm{v}), E_{22}=E_{2}(c)-E_{2}(\mathrm{v}), \ldots$. Os valores das energias de absorção são usados para se estudar os efeitos da funcionalização nas propriedades eletrônicas dos nanotubos. A dispersão de nanotubos em surfactantes possibilitou um estudo detalhado das propriedades óticas dos nanotubos e a correlação com a estrutura atômica ${ }^{28,29}$. Quando envoltos por micelas, os nanotubos semicondutores emitem luz cujo comprimento de onda está principalmente no infravermelho próximo. O espectro de emissão (fotoluminescência) é associado com a separação em energia dos dois primeiros picos $\left(\mathrm{E}_{11}\right)$, sendo observado apenas em nanotubos semicondutores. Essa técnica é muito importante para o estudo de nanotubos funcionalizados, pois pequenas mudanças no meio produzem efeitos mensuráveis no espectro de emissão de luz.

\section{FUNCIONALIZAÇÃO DE NANOTUBOS DE CARBONO}

Esta seção trata das alterações nas propriedades eletrônicas e estruturais de nanotubos de carbono, resultantes das diferentes formas de funcionalização. Neste caso, átomos ou moléculas podem ser adsorvidos ou ligados aos nanotubos alterando, de alguma forma, as propriedades originais dos nanotubos, tornando-os interessantes e com novas propriedades.

Experimentos relacionados com funcionalização de nanotubos tiveram início com a adsorção de flúor em SWNT e substituição de SWNT fluorados em solução ${ }^{30,31}$. Também tem sido intensamente investigada, tanto teórica como experimentalmente, a fun-cionalização de nanotubos de carbono através de suas paredes com a adsorção de átomos ou moléculas ${ }^{31-38}$, através de dopagens substitucionais dos tubos $^{39-41}$, por meio de deformações estruturais ${ }^{42,43}$ ou ainda por adsorção de grupos químicos, como o $\mathrm{COOH}^{44-46}$. Na maioria destes casos, as propriedades eletrônicas e, conseqüentemente, a reatividade química são alteradas em função da funcionalização. A seguir, descrevem-se alguns exemplos de funcionalização de nanotubos de carbono através de interações não-covalentes (sistemas que interagem fracamente com os nanotubos) e covalentes (sistemas que interagem fortemente com os nanotubos de carbono, gerando modificações drásticas nas suas propriedades).

\section{Interações não-covalentes}

Uma forma de analisar a interação dos nanotubos de carbono com átomos e moléculas é através do comportamento da transferência de carga entre o SWNT e o sistema adsorvido. Diversas técnicas 
experimentais podem ser usadas com essa finalidade. A técnica de espectroscopia Raman ressonante é muito poderosa nessa análise, pois se pode determinar esse processo através das mudanças induzidas no espectro Raman ressonante (intensidade, deslocamento nos números de onda e perfil dos picos) $)^{47}$. Em condições ressonantes as vibrações (fônons) estão acopladas com a estrutura eletrônica e os efeitos das interações não-covalentes na estrutura eletrônica dos nanotubos são transmitidos nas características espectrais. A Figura 4b mostra os espectros Raman de nanotubos de carbono puros e interagindo com $\mathrm{Ag}^{48}$.

(a)

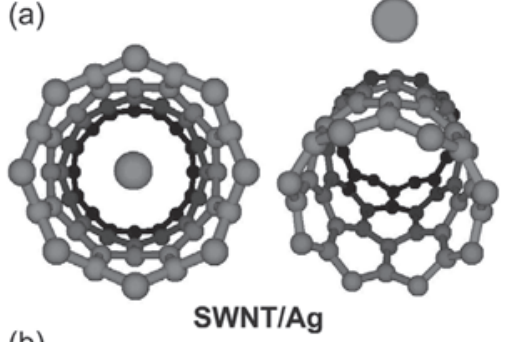

(b)

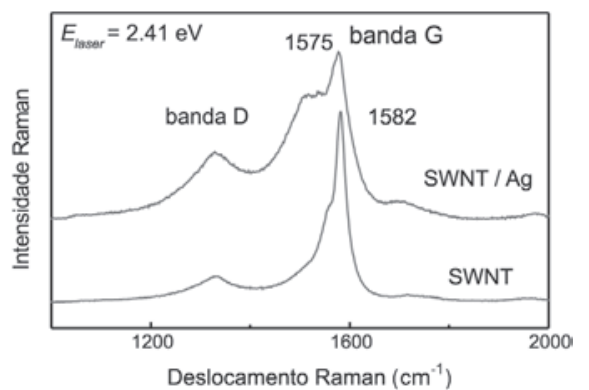

Figura 4. (a) Ilustração do nanotubo interagindo com Ag, tornando-o um sistema aceitador de carga; (b) espectros Raman dos nanotubos não modificados e interagindo com Ag. Reproduzida da ref. 37, com permissão da Elsevier

Na Figura 4b apresentam-se os espectros Raman ressonantes medidos com excitação $2,41 \mathrm{eV}(514,5 \mathrm{~nm})$. Para essa amostra de nanotubos antes da dopagem, em particular, esta linha de laser excita os nanotubos semicondutores e o perfil observado para a banda $\mathrm{G}$ é típico de nanotubos semicondutores. O espectro Raman dos nanotubos interagindo com $\mathrm{Ag}$ apresenta duas mudanças: um aumento acentuado na assimetria da banda $G$ e uma diminuição no número de onda dos modos vibracionais. O perfil da banda $\mathrm{G}$ (assimétrica e larga) observada no sistema SWNT/Ag é típico de nanotubos metálicos ${ }^{26}$. Esse perfil (chamado de "Breit-Wigner-Fano - BWF") é atribuído à interação das excitações dos elétrons livres dos nanotubos metálicos com os fônons dos nanotubos. Como a energia do laser de excitação foi mantida, os nanotubos excitados na amostra pura e adsorvida com Ag são os mesmos. O perfil BWF aparece porque os nanotubos semicondutores receberam carga da $\mathrm{Ag}$ e agora possuem portadores na banda de condução. Essa interpretação dos experimentos é suportada por resultados teóricos (cálculos $a b$ initio). Os estudos mostraram que a Ag interagindo com nanotubos de carbono se comporta como doadora de carga ${ }^{37}$. Os resultados teóricos também revelaram que as energias de ligações são baixas indicando que se trata de um processo de adsorção física e não de uma ligação química propriamente dita. Comportamentos similares ao descrito acima para Ag têm sido observados para nanotubos interagindo com metais alcalinos ${ }^{49,50}$. No grafite é observado que para moléculas doadoras (receptoras) a freqüência da banda $\mathrm{G}$ diminui (aumenta) à medida que a concentração das espécies aumenta. Vale salientar que nos nanotubos de carbono esse compor- tamento, para baixas concentrações de dopantes, é anômalo, conforme observado recentemente em nanotubos intercalados com $\mathrm{Rb}$ e $\mathrm{Cs}^{50,51}$. Esse comportamento pode estar associado às diferentes variações do comprimento de ligação $\mathrm{C}-\mathrm{C}$ ao longo do eixo do tubo e ao longo da circunferência, quando o nanotubo está dopado ${ }^{52}$.

Semelhante à química de intercalação do grafite, os nanotubos de carbono também têm sido intercalados com espécies receptoras, tais como os elementos halogênios ${ }^{49,53,54}$. Estudos de espectroscopia Raman ressonante mostram que nanotubos interagindo com $\mathrm{I}_{2} \mathrm{e}$ $\mathrm{Br}_{2}$ atuam como doadores de carga para essas moléculas ${ }^{49}$. O estudo destes sistemas de nanotubos interagindo com moléculas doadoras e receptoras é importante para o entendimento das propriedades dos nanotubos. Um estudo recente envolvendo nanotubos de paredes duplas (DWNT) interagindo com $\mathrm{Br}_{2}$ mostrou que a dopagem modifica os valores das transições eletrônicas dos nanotubos, deslocando-as para altas energias para alguns tubos e para mais baixas energias para outros ${ }^{55}$. Esse estudo também revelou que os nanotubos metálicos são mais sensíveis à presença do $\mathrm{Br}_{2}$ que os semicondutores. Esse sistema é interessante porque é possível observar também o espectro das moléculas de $\mathrm{Br}_{2}$ e identificar os picos no espectro Raman relacionado com o dopante (Figura 5$)^{55}$.
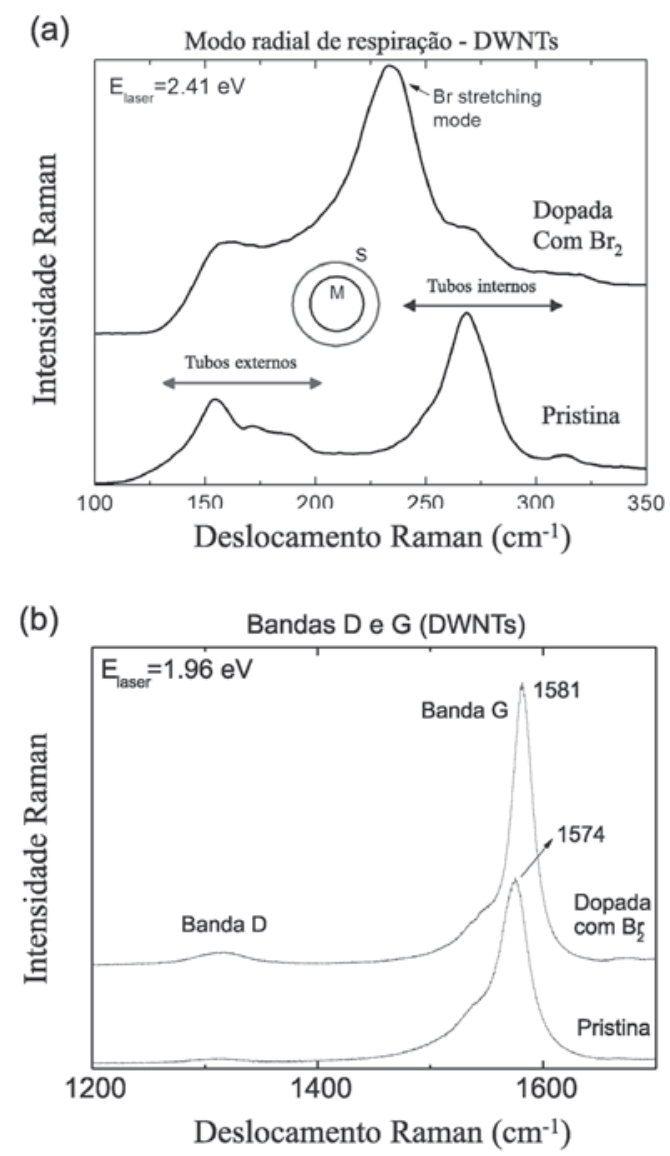

Figura 5. Espectros Raman de nanotubos de paredes duplas puros e intercalados com $\mathrm{Br}_{2}$ na região do modo radial de respiração (a) e na região da banda $G(b)$. Reproduzidos da ref. 55, com permissão da American Physical Society

Nanotubos de carbono também tem sido propostos como materiais eficientes para remoção de poluentes, tais como diclorobenzeno ${ }^{56}$ e dioxina ${ }^{57}$. Mostra-se através de resultados experimentais $^{57}$ e teóricos ${ }^{58}$ que os nanotubos de carbono são capazes de remover dioxinas de ambientes através da interação entre os anéis da dioxina com os anéis do tubo, o que faz com que, inclusive, a 
molécula que era originalmente planar, sofra distorções, como mostra a Figura 6. Nanotubos de carbono sem defeitos possuem uma interação fraca com a dioxina (Figuras $6 \mathrm{a}$ e b), mas quando a estrutura apresenta defeitos ocorre uma interação muito mais forte com energia de ligação típica de uma ligação química (Figuras $6 \mathrm{c}$ e d) ${ }^{58}$. Comportamento semelhante foi observado para o 1,2-diclorobenzeno, cuja adsorção nas paredes dos nanotubos ocorre via interações do tipo empilhamento $\pi$ ( $\pi$-“stacking") $)^{45}$.
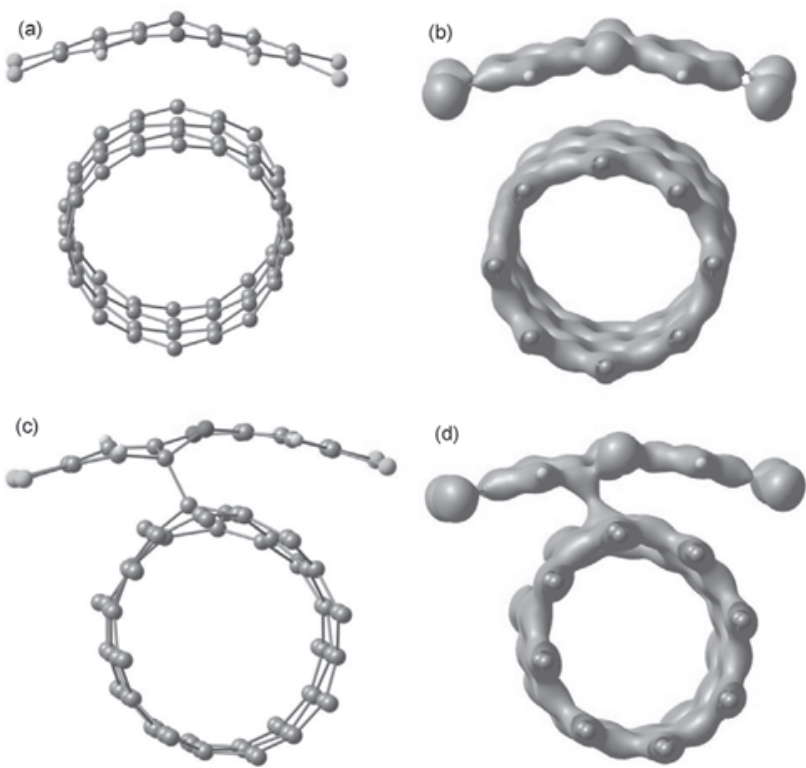

Figura 6. Em (a) e (c) apresenta-se a configuração estrutural mais estável obtida, através de cálculos ab initio, para a dioxina interagindo com um SWNT $(8,0)$ perfeito e com defeitos estruturais, respectivamente; em (b) e (d), o "contour plot" da densidade de carga eletrônica total dos sistemas (a) $e(c)$, correspondentes ${ }^{58}$

Uma das principais vantagens da funcionalização não-covalente é que as propriedades eletrônicas dos nanotubos são mantidas porque a estrutura $s p^{2}$ e a conjugação dos átomos de carbono do tubo são conservadas. Um exemplo ilustrativo do uso dessa característica da funcionalização não-covalente é a solubilização dos nanotubos de carbono, envolvendo-os com cadeias poliméricas, tais como dodecil sulfato de sódio (SDS), polivinil, poliestireno, entre outros $28,29,59,60$. A região hidrofóbica dessas moléculas interage com a superfície dos nanotubos, destruindo a interface hidrofóbica dos nanotubos com a água e a interação tubo-tubo (van der Waals) responsável pela agregação em feixes. As micelas formadas por surfactante e nanotubos formam dispersões estáveis em meio aquoso. As miscelas mantêm os nanotubos de carbono isolados uns dos outros, permitindo o estudo das propriedades fotofísicas (absorção, emissão, "excitons") de cada nanotubo e a correlação dessas propriedades físicas com a estrutura atômica, ou seja, com os índices $n$ e $m^{61-63}$. A interação dos SWNTs com seqüências de oligonucleotídeos (Figura 7) tem sido um dos métodos mais utilizados e com maior sucesso para a separação de nanotubos metálicos de nanotubos semicondutores e, também, na separação de nanotubos pelo diâmetro e comprimento ${ }^{63,64}$. Os nanotubos semicondutores e metálicos possuem diferentes valores de constante dielétrica e portanto interagem de forma diferente com as sequiências de oligonucleotídeos. O resultado é que em uma coluna de cromatografia de troca iônica eles ficam retidos em diferentes posições, permitindo extrair frações de amostras enriquecidas com nanotubos metálicos e outras frações enriquecidas com nanotubos semicondutores ${ }^{63}$. (a)

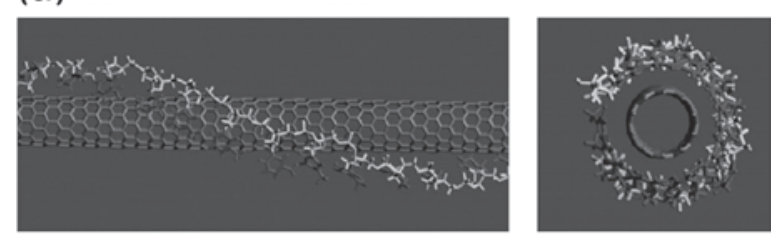

(b)

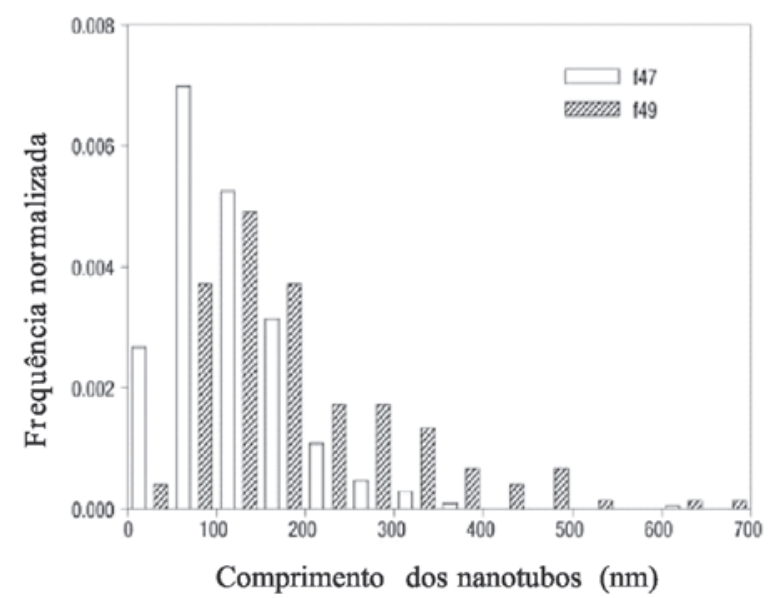

Figura 7. - (a) Mostra o encapsulamento de um nanotubo por DNA. (b) Distribuição do comprimento dos nanotubos para diferentes frações separadas na coluna de cromatografia de troca iônica. Reproduzida da ref. 63, com permissão da Macmillan Publishers Ltd: Nature Materials

\section{Interações covalentes}

Os nanotubos de carbono possuem superfícies com alta estabilidade química, portanto, poucos átomos e moléculas podem interagir diretamente com suas paredes. A maioria dos experimentos realizados para funcionalizar os nanotubos de carbono são sistemas muito reativos. Ao contrário dos fulerenos, a reatividade química dos nanotubos é dominada pelo desemparelhamento dos orbitais $\pi$ entre os carbonos adjacentes na superfície curva do nanotubo. Nos fulerenos a reatividade é devida ao ângulo de piramidalização dos orbitais $p^{65}$. Apesar da química de nanotubos ter sido desenvolvida somente nos últimos anos, já existe uma grande variedade de rotas e estratégias químicas usadas para funcionalizar covalentemente os nanotubos. A seguir serão discutidos apenas alguns exemplos.

O primeiro exemplo a ser discutido é a interação dos nanotubos com um agente fortemente oxidante, o $\mathrm{CrO}_{3}{ }^{37}$. Na Figura 8 mostra uma situação oposta ao que foi discutido anteriormente para os nanotubos interagindo com Ag. Para a amostra analisada, Figura 8, os nanotubos puros excitados com a linha de laser 1,96 eV (633 $\mathrm{nm}$ ) são metálicos, como indicado pelo perfil da banda G. Quando os nanotubos interagem com $\mathrm{CrO}_{3}$ pode-se observar algumas mudanças no espectro Raman. O perfil BWF da banda G muda para um perfil típico de nanotubos semicondutores e os números de onda aumentam. A destruição do perfil BWF após a adsorção é devida à remoção dos elétrons livres do sistema metálico, diminuindo a interação dos elétrons com os fônons

Alguns metais de transição, como Ti, Pd, Mn, Fe etc., mostram-se como elementos com grande capacidade de adsorver nas paredes dos SWNTs, o que é visto no trabalho de Zhang e colaboradores $^{38}$, que recobriram nanotubos de carbono com metais de transição, como Ti, Ni, Pd, Au, Al e Fe (Figura 9a-f, respectivamente). 
(a)

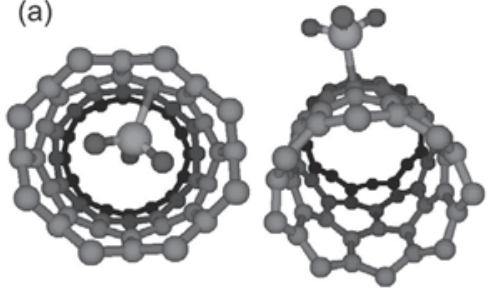

$\mathrm{SWNT} / \mathrm{CrO}_{3}$

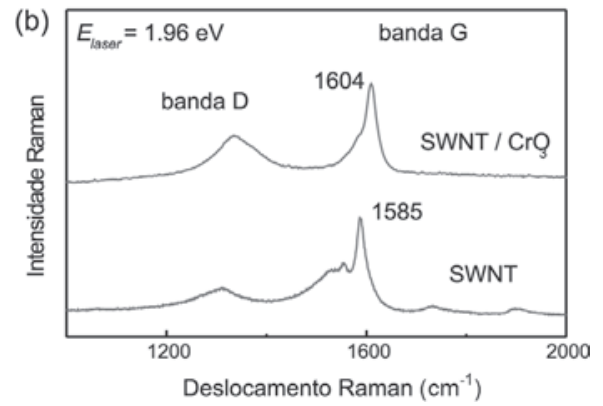

Figura 8. (a) Ilustração dos nanotubos interagindo com $\mathrm{CrO}_{3}$, tornando o tubo um sistema doador de carga; (b) espectros Raman dos nanotubos não modificados e interagindo com $\mathrm{CrO}_{3}$. Reproduzidos da ref. 37, com permissão da Elsevier
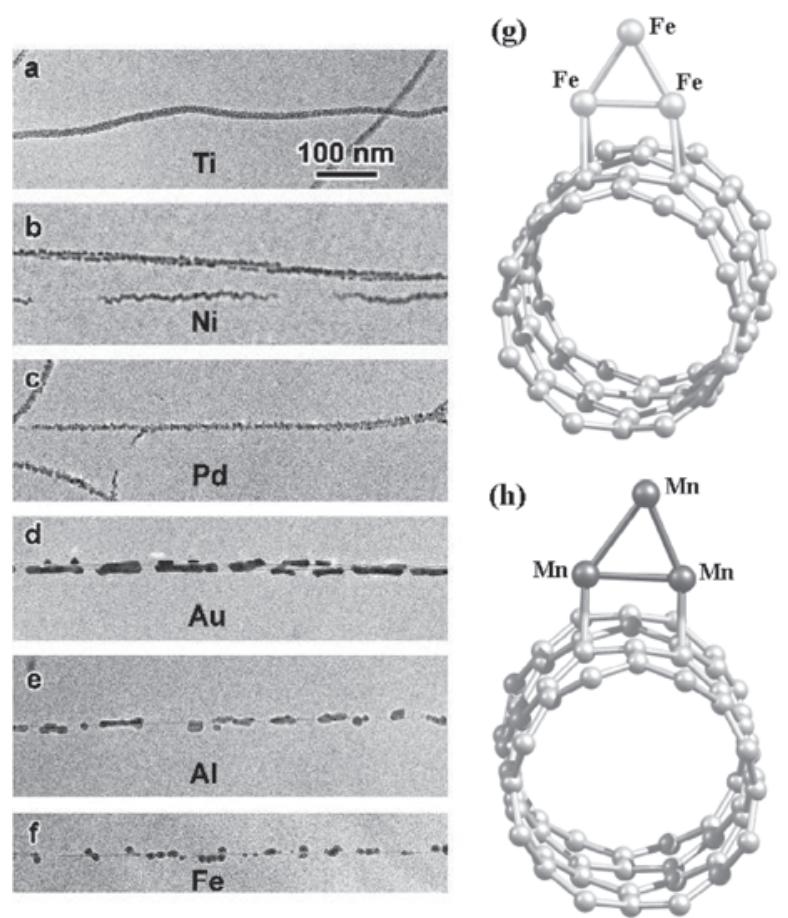

Figura 9. (a)-(f) Imagem de microscopia eletrônica de transmissão de nanotubos de carbono recobertos com Ti, Ni, Pd, Au, Al e Fe. As figuras ( $(g) e$ (h) correspondem a estruturas otimizadas através de simulações para clusters de Fe e Mn, respectivamente. Reproduzidos da ref. 38, com permissão da Elsevier

Nas Figuras 9g e h apresentam-se simulações através de métodos de química quântica ( $a b$ initio) usando nanotubos de carbono interagindo com pequenos aglomerados de $\mathrm{Fe}$ e $\mathrm{Mn}$, mostrando a capacidade destes metais adsorverem fortemente nos tubos. Utilizando-se nanotubos cobertos com metais de transição podem ser anexados átomos ou moléculas não interagentes diretamente com o tubo e que agora podem vir a interagir com os tubos funcionalizados com estes metais. Desta forma, abre-se a possibilidade de seu uso como armazenadores de combustível, por meio de possíveis rotas para armazenagem de $\mathrm{H}_{2}$ ou também como removedores de moléculas tóxicas, como $\mathrm{CO}, \mathrm{CO}_{2}$ e $\mathrm{SO}_{2}{ }^{36}$.

A funcionalização química de nanotubos também tem sido intensamente analisada de forma a se anexar à superfície do tubo grupos químicos através de ligações covalentes. Entre os vários grupos usados para funcionalização destaca-se o grupo carboxílico considerado grupo padrão para este tipo de proposta ${ }^{42,66-68}$. Este fato é devido ao átomo de carbono do $\mathrm{COOH}$ ligar-se covalentemente com os $\mathrm{C}$ do tubo, tornando fácil a remoção do grupo $\mathrm{OH}$ usando um agente acoplador (cloreto de tionila, por ex.), podendo-se anexar outros grupos ou moléculas. A Figura 10 mostra uma rota usada para incorporação de moléculas de $\mathrm{NH}_{2}$ em nanotubos de carbono através de reações com grupos carboxílicos ${ }^{44}$.

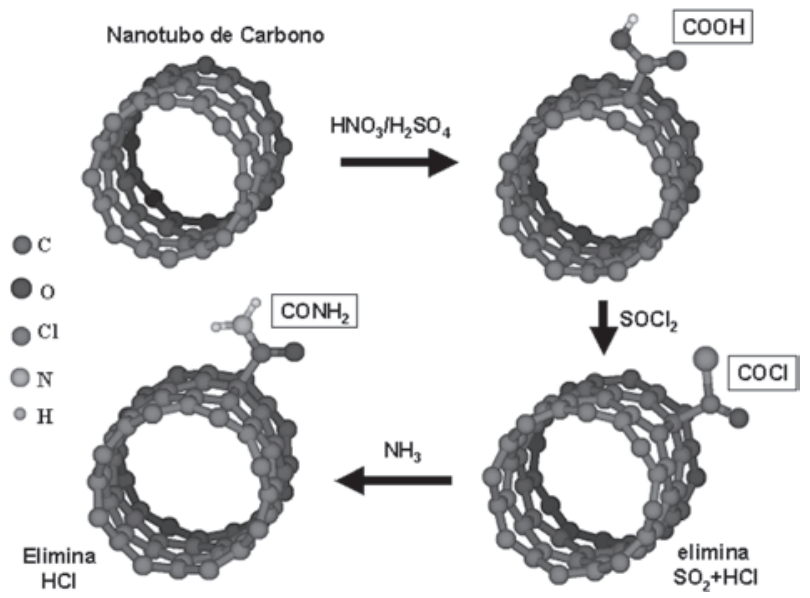

Figura 10. Rota química usada para anexar grupos $\mathrm{COOH}$ em nanotubos de carbono e subseqüentemente converter em outros radicais desejados, como o $\mathrm{CO}-\mathrm{NH}_{2}$

Este tipo de interação através de grupos carboxílicos abre possibilidades muito interessantes para aportar moléculas mais complexas como aminoácidos, DNA e marcadores fluorescentes ${ }^{68,69}$. Ramanathan e colaboradores ${ }^{67}$ anexaram grupos amida e amina em nanotubos de carbono funcionalizados com $\mathrm{COOH}$, seguindo o esquema da Figura 10.

Uma outra forma de funcionalização covalente é através da dopagem substitucional de átomos. Os exemplos mais típicos desse tipo de funcionalização são os nanotubos de carbono dopados com $\mathrm{N}^{70}, \mathrm{~B}^{71-73}$, ou ambos ${ }^{74,75}$. Esses sistemas dopados são interessantes, pois aumentam significativamente a reatividade dos nanotubos. Desta forma, a dopagem substitucional torna-se altamente interessante. A Figura 11 apresenta o exemplo do aminoácido alanina que não adsorve no nanotubo de carbono, mas que mostra uma grande interação através de uma dopagem de $\mathrm{B}$ do nanotubo ${ }^{76}$. De acordo com os cálculos ab initio, os nanotubos de carbono dopados com B podem interagir diretamente com aminoácidos gerando sistemas híbridos tais como SWNT-aminoácidos.

As principais rotas químicas utilizadas na funcionalização de nanotubos podem ser resumidas na Figura 12. Essas rotas químicas tem sido utilizadas com sucesso para modificar a superfície dos nanotubos e obter sistemas auto-organizados. Para uma descrição detalhada das diversas reações químicas ver ref. 77 .

\section{APLICAÇÕES DE NANOTUBOS FUNCIONALIZADOS}

Nanotubos de carbonos do tipo MWNT, DWNT e SWNT quando funcionalizados (covalente e não-covalente) possuem uma ri- 

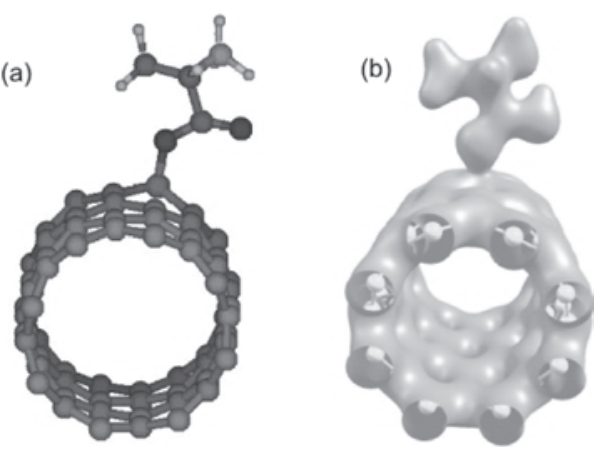

Figura 11. (a) Configuração estrutural da alanina interagindo com um SWNT dopado com B; (b) plot da distribuição total de carga indicando a forte interação da molécula com o nanotubo dopado

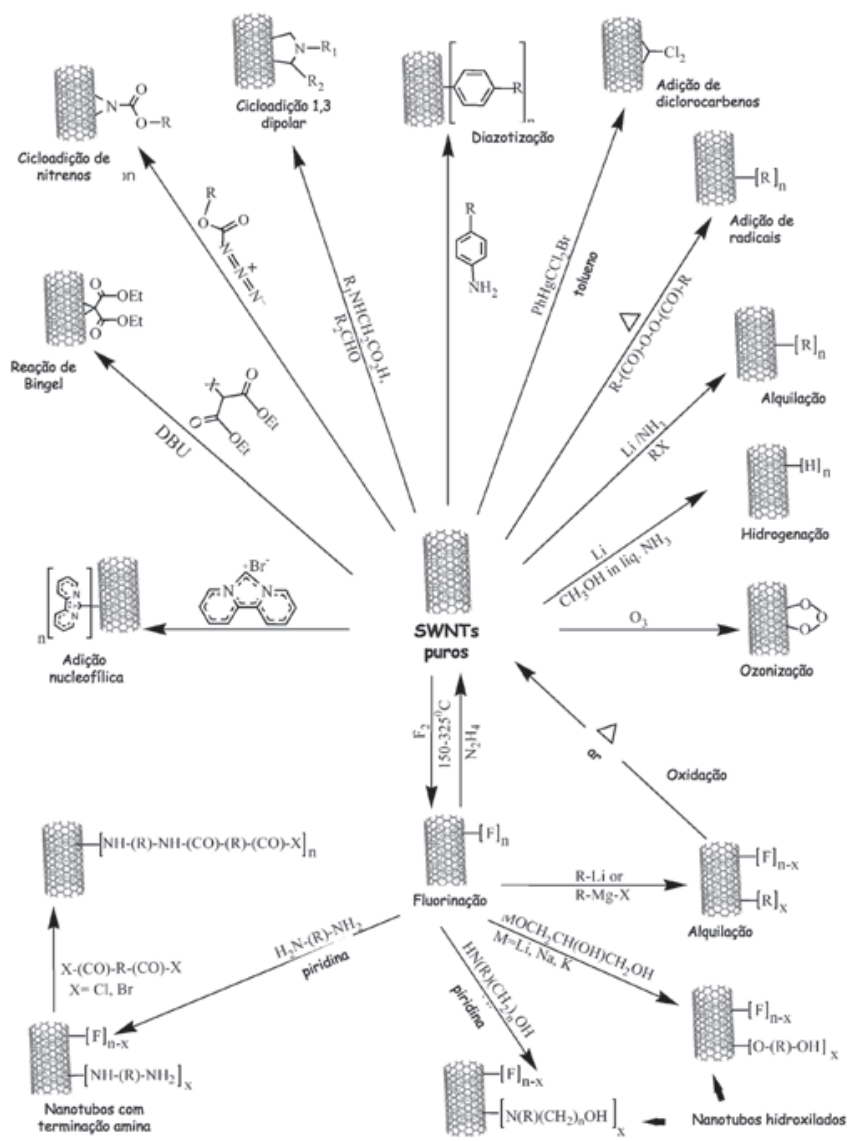

Figura 12. Principais rotas químicas utilizadas na funcionalização covalente de nanotubos de carbono. Reproduzido da ref. 77, com permissão da Wiley

queza muito grande em termos de propriedades físico-químicas e têm sido usados nos mais engenhosos mecanismos para produzir sistemas nanoestruturados com arquiteturas complexas e com funções altamente especializadas.

É possível alinhar nanotubos de carbono sobre uma superfície previamente recoberta com DNA dispostos em padrões pré-determinados. As fitas de DNA sobre os substratos são decoradas com 1pirenoetilamina hidroclorídrica (PMA) ${ }^{78}$. Uma gota de nanotubos em suspensão é colocada em contato com o substrato e o resultado são nanotubos alinhados sobre o substrato. $\mathrm{O}$ grupo amina (carregado positivamente) do PMA interage com o grupo fosfato (carregado negativamente) das fitas de DNA. O grupo pirenil, por sua vez, interage com os anéis dos nanotubos através do empilhamento $\pi$ ( $\pi$-“stacking"). O resultado dessas interações é que os nanotubos ficam alinhados seguindo o padrão com que as fitas de DNA foram dispostas no substrato. Os nanotubos de carbono dopados com $\mathrm{N}\left(\mathrm{CN}_{\mathrm{x}}\right)$ têm sido usados para imobilizar as proteínas ferritina e albumina (BSA) ${ }^{79}$.

Uma das áreas mais promissoras da nanotecnologia em geral, envolvendo cifras consideráveis, é a área de nanocompósitos. Os nanotubos de carbono possuem um papel muito importante na confecção de compósitos, geralmente usando nanotubos dispersos em polímeros ${ }^{75-77,80-83}$. Nanotubos de carbono são usados como elementos de reforço e também como elementos fundamentais para a nova geração de tecidos têxteis, os chamados tecidos eletrônicos ${ }^{78}$.

Muitas aplicações de nanotubos funcionalizados, que inclusive já estão chegando no mercado, são o seu uso como sensores biológicos implantáveis no corpo humano. Um exemplo é o monitoramento contínuo dos níveis de glucose. O biossensor explora a propriedade de fotoluminescência dos nanotubos semicondutores quando esses estão dispersos. Uma reação enzimática converte a glucose em gluconolactana cujo sub-produto (peróxido de hidrogênio) interage com grupos $\mathrm{Fe}(\mathrm{CN})_{6}^{3-}$ que estão anexados às paredes dos nanotubos. Os nanotubos são encapsulados em um vidro com uma capilaridade tal que permite a entrada da glucose e evita o contato com células do corpo. A potência do brilho do sensor, iluminado por laser no infravermelho, é diretamente relacionada com a concentração de glucose nos tecidos ${ }^{84}$.

Os nanotubos de carbono envoltos por DNA foram recentemente colocados no interior de células. Monitorando-se as propriedades óticas demonstrou-se que é possível detectar concentrações em níveis moleculares de substâncias ${ }^{85}$. O DNA na presença de alguns íons $\left(\mathrm{Hg}^{+}\right.$, por ex.) sofre uma mudança na configuração, reduzindo a área que recobre o nanotubo. A conseqüência desse efeito é o decréscimo nas energias de emissão de cada nanotubo. Essa proposta de biossensor é muito promissora na área de biomarcadores. A principal vantagem desse sistema é o fato dos nanotubos apresentarem fluorescência no infravermelho próximo, onde a maioria dos tecidos celulares são transparentes. Exemplos como estes ilustram o enorme potencial de aplicação dos nanotubos de carbono funcionalizados.

\section{COMENTÁRIOS FINAIS}

A funcionalização de nanotubos de carbono é um tema muito rico para pesquisa básica, permitindo o entendimento mais aprofundado das propriedades físico-químicas dos nanotubos e também das espécies funcionais como foi discutido através de alguns exemplos neste artigo. As características únicas dos nanotubos aliadas ao desenvolvimento da química destes sistemas têm despontado com um número considerável de aplicações e promessas revolucionárias em diversas áreas de atividade humana, tais como medicina, farmacologia, eletrônica, computação, robótica, indústria química, petroquímica e meio ambiente.

Devido à propriedade de biocompatibilidade dos nanotubos de carbono, o potencial de aplicação na área biomédica é enorme, principalmente nas áreas de engenharia de tecidos, biocompósitos e transfecção genética. $\mathrm{O}$ entendimento básico, a descrição e o controle dos sistemas nano-bio levará a uma nova geração de sistemas integrados, que combinam as propriedades peculiares dos nanotubos de carbono com a capacidade de reconhecimento molecular das moléculas biológicas. Essas são áreas que necessitam um esforço inter/multidisciplinar muito forte e é uma boa oportunidade para o Brasil, pois o país possui competências estabelecidas na síntese e caracterização de nanotubos (existe uma rede nacional de pesquisa em nanotubos de carbono $^{86}$ e um instituto do Milênio de Nanotecnologia que também aborda esse tema) e também possui excelência em genética (Programa Genoma Brasileiro). 
Na concepção dos autores, essa área nano-bio é muito importante para o desenvolvimento da nanociência no Brasil e contribui para a consolidação das áreas de fronteira, inclusive formando recursos humanos, desde a iniciação científica até a pós-graduação, com fortes componentes de multidisciplinaridade na formação em contraposição às formações puramente clássicas. Essa abordagem é um ingrediente fundamental para termos, no futuro, uma massa crítica de pesquisadores preparada para as revoluções tecnológicas decorrentes da Nanociência.

\section{AGRADECIMENTOS}

Aos diversos colaboradores, no Brasil e no exterior, pelas discussões e oportunidades de colaboração científica no estudo dos nanotubos de carbono. Agradecemos o financiamento da rede Nacional de Pesquisa em Nanotubos de Carbono, do Instituto do Milênio de Nanotecnologia, do Instituto do Milênio de Materiais Complexos, e da Rede de Pesquisa em Nanobioestruturas (MCT-CNPq). Às agências financiadoras CNPq, FUNCAP, FAPERGS e CAPES pelo apoio financeiro. S. B. Fagan agradece à L'oreal pelo Prêmio L'Oreal Brasil de Mulheres na Ciência.

\section{REFERÊNCIAS}

1. Kroto, H. W.; Heath, J. R.; O’Brien, S. C.; Curl, R. F.; Smalley, R. E.; Nature 1985, 318, 162.

2. Iijima, S.; Nature 1991, 354, 56.

3. Iijima, S.; Ichihashi, T.; Nature 1993, 363, 603

4. Bethune, D. S.; Kiang, C. H.; Devries, M. S.; Gorman, G.; Savoy, R.; Vazquez, J.; Beyers, R.; Nature 1993, 363, 605.

5. Monthioux, M.; Kuznetsov, V. L.; Carbon 2006, 44, 1621.

6. Thess, A.; Lee, R.; Nikolaev, P.; Dai, H. J.; Petit, P.; Robert, J.; Xu, C. H.; Lee, Y. H.; Kim, S. G.; Rinzler, A. G.; Colbert, D. T.; Scuseria, G. E.; Tomanek, D.; Fischer, J. E.; Smalley, R. E.; Science 1996, 273, 483.

7. Odom, T. W.; Huang, J. L.; Kim, P.; Lieber, C. M.; Nature 1998, 391, 62.

8. Wildoer, J. G. W.; Venema, L. C.; Rinzler, A. G.; Smalley, R. E.; Dekker, C.; Nature 1998, 391, 59.

9. Rao, A. M.; Richter, E.; Bandow, S.; Chase, B.; Eklund, P. C.; Williams, K. W.; Fang, S.; Subbaswamy, K. R.; Menon, M.; Thess, A.; Smalley, R. E.; Dresselhaus, G.; Dresselhaus, M. S.; Science 1997, 275, 187.

10. Saito, R.; Fujita, M.; Dresselhaus, G.; Dresselhaus, M. S.; Appl. Phys. Lett. 1992, 60, 2204.

11. Mintmire, J. W.; Dunlap, B. I.; White, C. T.; Phys. Rev. Lett. 1992, 68, 631.

12. Liu, J.; Fan, S.; Dai, H.; Mater. Res. Bull. 2004, 224.

13. Dresselhaus, M. S.; Dresselhaus, G.; Avouris, P.; Carbon Nanotubes: Synthesis, Structure, Properties, and Applications Topics in Applied Physics, Springer-Verlag: New York, 2001, vol. 80.

14. Dai, H. J.; Surf. Sci. 2002, 500, 218.

15. Fu, K. F.; Huang, W. J.; Lin, Y.; Zhang, D. H.; Hanks, T. W.; Rao A. M.; Sun, Y. P.; J. Nanosci. Nanotechnol. 2002, 2, 457.

16. Kong, J.; Franklin, N. R.; Zhou, C.; Chapline, M. G.; Peng, S.; Cho K.; Dai, H.; Nature 2000, 287, 622.

17. Li, J.; Lu, Y.; Cinke, M.; Han, J.; Meyyappan, M.; Nano Lett. 2003, 3, 929.

18. da Silva, L. B.; Fagan, S. B.; Mota, R.; Nano Lett. 2004, 4, 65.

19. Sinnott, S. B.; J. Nanosci. Nanotechnol. 2002, 2, 113.

20. Srivastava, A.; Srivastava, O. N.; Talapatra, S.; Vajtai, R.; Ajayan, P. M.; Nat. Mater. 2004, 3, 610.

21. Hirsch, A.; Angew. Chem., Int. Ed. 2002, 41, 1853.

22. Saito, R.; Dresselhaus, G.; Dresselhaus, M. S.; Physical Properties of Carbon Nanotubes, Imperial College Press: London, 1998.

23. Dresselhaus, M. S.; Dresselhaus, G.; Jorio, A.; Souza Filho, A. G.; Saito, R.; Carbon 2002, 40, 2043.

24. Barros, E. B.; Jorio, A.; Samsonidze, G. G.; Capaz, R. B.; Souza Filho, A. G.; Mendes Filho, J.; Dresselhaus, G.; Dresselhaus, M. S.; Phys. Reports 2006, 431, 261.

25. Jorio, A.; Pimenta, M. A.; Souza Filho, A. G.; Saito, R.; Dresselhaus, M. S.; Dresselhaus, G.; New J. Phys. 2003, 5, 137.

26. Pimenta, M. A.; Marucci, A.; Empedocles, S.; Bawendi, M.; Hanlon, E. B.; Rao, A. M.; Eklund, P. C.; Smalley, R. E.; Dresselhaus, G.; Dresselhaus, M. S.; Phys. Rev. B: Condens. Matter Mater. Phys. 1998, 58, 16016.

27. Souza Filho, A. G.; Jorio, A.; Dresselhaus, G.; Dresselhaus, M. S.; Saito, R.; Swan, A. K.; Unlu, M. S.; Goldberg, B. B.; Hafner, J. H.; Lieber, C.
M.; Pimenta, M. A.; Phys. Rev. B: Condens. Matter Mater. Phys. 2002, 65,035404

28. O’Connell, M. J.; Bachilo, S. M.; Huffman, C. B.; Moore, V. C.; Strano, M. S.; Haroz, E. H.; Rialon, K. L.; Boul, P. J.; Noon, W. H.; Kittrell, C.; Ma, J. P.; Hauge, R. H.; Weisman, R. B.; Smalley R. E.; Science 2002, 297, 593.

29. Bachilo, S. M.; Strano, M. S.; Kittrell, C.; Hauge, R. H.; Smalley, R. E.; Weisman, R. B.; Science 2002, 298, 2361.

30. Mickelson, E. T.; Huffman, C. B.; Rinzler, A. G.; Smalley, R. E., Hauge, R. H.; Margrave, J. L.; Chem. Phys. Lett. 1998, 296, 188.

31. Mickelson, E. T.; Huffman, C. B.; Rinzler, A. G.; Smalley, R. E.: Hauge, R. H.; Margrave, J. L.; J. Phys.Chem. B 1999, 103, 4318.

32. Fagan, S. B.; Mota, R.; da Silva, A. J. R.; Fazzio, A.; Phys. Rev. B: Condens. Matter Mater. Phys. 2003, 67, 205414.

33. Kukovecz, A.; Pichler, T.; Pfeiffer, R.; Kramberger, C.; Kuzmany, H.; Phys. Chem. Chem. Phys. 2003, 5, 582.

34. Fagan, S. B.; Mota, R.; da Silva, A. J. R.; Fazzio, A.; J. Phys.: Condens. Matter. 2004, 16, 3647.

35. Peng, S.; Cho, K.; Nanotechnology 2000, 11, 57.

36. Yildirim, T.; Ciraci, S.; Phys. Rev. Lett. 2005, 94, 175501.

37. Fagan, S. B.; Souza Filho, A. G.; Mendes Filho, J.; Corio, P.; Dresselhaus, M. S.; Chem. Phys. Lett. 2005, 406, 59.

38. Zhang, Y.; Franklin, N. W.; Chen, R. J.; Dai, H. J.; Chem. Phys. Lett. 2000, $331,35$.

39. Choi, H. J.; Ihm, J.; Louie, S. G.; Cohen, M. L.; Phys. Rev. Lett. 2000, 84, 2917.

40. Baierle, R. J.; Fagan, S. B.; Mota, R.; da Silva, A. J. R.; Fazzio, A.; Phys. Rev. B: Condens. Matter Mater. Phys. 2001, 64, 85413.

41. Fagan, S. B.; Baierle, R. J.; Mota, R.; da Silva, A. J. R.; Fazzio, A.; Phys. Rev. B: Condens. Matter Mater. Phys. 2003, 67, 33405.

42. Zhao, J.; Park, H.; Han, J.; Lu, J. P.; J. Phys. Chem. B 2004, 108, 4227.

43. Fagan, S. B.; da Silva, L. B.; Mota, R.; Nano Lett. 2003, 3, 289.

44. Veloso, M. V.; Souza Filho, A. G.; Mendes Filho, J.; Fagan, Solange B.; Mota, R.; Chem. Phys. Lett. 2006, 430, 71.

45. Fagan, S. B.; Souza Filho, A. G.; Lima, J. O. G.; Mendes Filho, J.; Ferreira, O. P.; Mazali, I. O.; Alves, O. L.; Dresselhaus, M. S.; Nano Lett. 2004, 4, 1285.

46. Fagan, S. B.; Mota, R.; da Silva, A. J. R.; Fazzio, A.; Nano Lett. 2004, 4, 975.

47. Souza Filho, A. G.; Jorio, A.; Samsonidze, G. G.; Dresselhaus, G.; Saito, R.; Dresselhaus, M. S.; Nanotechnology 2003, 14, 1130.

48. Corio, P.; Santos, A. P.; Santos, P. S.; Temperini, M. L. A.; Brar, V. W.; Pimenta, M. A.; Dresselhaus, M. S.; Chem. Phys. Lett. 2004, 383, 475.

49. Rao, A. M.; Eklund, P. C.; Bandow, S.; Thess, A.; Smalley, R. E.; Nature 1997, 388, 34; Shin, H. C.; Liu, M. L.; Sadanadan, B.; Rao, A. M.; J. Solid State Electrochem. 2004, 8, 908.

50. Chen, G. G.; Furtado, C. A.; Bandow, S.; Iijima, S.; Eklund, P. C.; Phys. Rev. B: Condens. Matter Mater. Phys. 2005, 71, 45408.

51. Chen, G.; Furtado, C. A.; Kim, U. J.; Eklund, P. C.; Phys. Rev. B: Condens. Matter Mater. Phys. 2005, 72, 155406.

52. Terrazos, L. A.; Capaz, R. B.; resultados não publicados.

53. Chen, G. G.; Bandow, S.; Margine, E. R.; Nisoli, C.; Kolmogorov, A. N.; Crespi, V. H.; Gupta, R.; Sumanasekera, G. U.; Iijima, S.; Eklund, P. C.; Phys. Rev. Lett. 2003, 90, 257403.

54. Cambedouzou, J.; Sauvajol, J. L.; Rahmani, A.; Flahaut, E.; Peigney, A.; Laurent, C.; Phys. Rev. B: Condens. Matter Mater. Phys. 2004, 69, 235422.

55. Souza Filho, A. G.; Endo, M.; Muramatsu, H.; Hayashi, T.; Kim, Y. A.; Barros, E. B.; Akuzawa, N.; Samsonidze, G. G.; Saito, R.; Dresselhaus, M. S.; Phys. Rev. B: Condens. Matter Mater. Phys. 2006, 73, 235413.

56. Peng, X.; Li, Y.; Luan, Z.; Di, Z.; Wang, H.; Tian, B.; Jia, Z.; Chem. Phys. Lett. 2003, 376, 154.

57. Long, R. Q.; Yang, R. T.; J. Am. Chem. Soc. 2001, 123, 2058.

58. Fagan, S. B.; Santos, E. J. G.; Souza Filho, A. G.; Mendes Filho, J.; Fazzio, A.; Chem. Phys. Lett. 2007, 437, 79.

59. Hill, D. E.; Lin, Y.; Rao, A. M.; Allard, L. F.; Sun, Y. P.; Macromolecules 2002, 35, 9466.

60. Lin, Y.; Rao, A. M.; Sadanadan, B.; Kenik, E. A.; Sun, Y. P.; J. Am. Chem. Soc. 2002, 106, 1294.

61. Chou, S. G.; Plentz, F.; Jiang, J.; Saito, R.; Nezich, D.; Ribeiro, H. B.; Jorio, A.; Pimenta, M. A.; Samsonidze, G. G.; Santos, A. P.; Zheng, M.; Onoa, G. B.; Semke, E. D.; Dresselhaus, G.; Dresselhaus, M. S.; Phys. Rev. Lett. 2005, 94, 127402.

62. Plentz, F.; Ribeiro, H. B.; Jorio, A.; Strano, M. S.; Pimenta, M. A.; Phys. Rev. Lett. 2005, 95, 247401.

63. Zheng, M.; Jagota, A.; Semke, E. D.; Diner, B. A.; Mclean, R. S.; Lustig, S. R.; Richardson, S. E.; Tassi, N. G.; Nat. Mater. 2003, 2, 338.

64. Zheng, M.; Jagota, A.; Strano, M. S.; Santos, A. P.; Barone, P.; Chou, S. G.; Diner, B. A.; Dresselhaus, M. S.; McLean, R. S.; Onoa, G. B.; Samsonidze, G. G.; Semke, E. D.; Usrey, M.; Walls, D. J.; Science 2003, $302,1545$. 
65. Haddon, R. C.; Acc. Chem. Res. 1998, 21, 243.

66. Chiu, P. W.; Duesberg, G. S.; Dettlaff-Weglikowska, U.; Roth, S.; Appl. Phys. Lett. 2002, 80, 3811.

67. Ramanathan, T.; Fischer, F. T.; Ruoff, R. S.; Brinson, L. C.; Chem. Mater 2005, 17, 1290 .

68. Kam, N. W. S.; Jessop, T. C.; Wender, P. A.; Dai, H.; J. Am. Chem. Soc. 2004, 126, 6850 .

69. Williams, K. A.; Veenhuizen, P. T. M.; de la Torre, B. G.; Eritja, R.; Dekker, C.; Nature 2002, 420, 761 .

70. Keskar, G.; Rao, R.; Luo, J.; Hudson, J.; Chen, J.; Rao, A. M.; Chem. Phys. Lett. 2005, 412, 269.

71. McGuire, K.; Gothard, N.; Gai, P. L.; Dresselhaus, M. S.; Sumanasekera, G.; Rao, A. M.; Carbon 2005, 3, 219.

72. Endo, M.; Muramatsu, H.; Hayashi, T.; Kim, Y. A.; van Lier, G.; Charlier J. C.; Terrones, H.; Terrones, M.; Dresselhaus, M. S.; Nano Lett. 2005, 5 1099

73. Terrones, M.; Small 2005, 1, 1032.

74. Terrones, M.; Golberg, D.; Grobert, N.; Seeger, T.; Reyes-Reyes, M.; Mayne, M.; Kamalakaran, R.; Dorozhkin, P.; Dong, Z. C.; Terrones, H.; Ruhle, M.; Bando, Y.; Adv. Mater. 2003, 15, 1899.

75. Terrones, M.; Grobert, N.; Terrones, H.; Carbon 2002, 40, 1665
76. Fagan, S. B.; resultados não publicados.

77. Banerjee, S.; Benny, H-. T.; Wong, S. S.; Adv. Mater. 2005, 17, 17; Sun, Y. P.; Fu, K. F.; Lin, Y.; Huang, W. J.; Acc. Chem. Res. 2002, 35, 1096; Niyogi, S.; Hamon, M. A.; Hu, H.; Zhao, B.; Bhowmik, P.; Sen, R.; Itkis, M. E.; Haddon, R. C.; Acc. Chem. Res. 2002, 35, 1105.

78. Xin, H.; Woolley, A. T.; J. Am. Chem. Soc. 2003, 125, 8710

79. Jiang, K. Y.; Schadler, L. S.; Siegel, R. W.; Zhang, X. J.; Zhang, H. F.; Terrones, M.; J. Mater. Chem. 2004, 14, 37.

80. Thostenson, E. T.; Ren, Z. F.; Chou, T. W.; Compos. Sci. Technol. 2001, 61, 1899.

81. Paiva, M. C.; Zhou, B.; Fernando, K. A. S.; Lin, Y.; Lopes, P. E.; Pennington, W. T.; Kennedy, J. M.; Sun, Y. P.; Compos. Interfaces 2005, $12,757$.

82. Do Nascimento, G. M.; Corio, P.; Novickis, R. W.; Temperini, M. L. A.; Dresselhaus M. S.; J. Polym. Sci., Part A: Polym. Chem. 2005, 43, 815.

83. Laxminarayana, K.; Jalili, N.; Text. Res. J. 2005, 75, 670.

84. Barone, P. W.; Baik, S.; Heller, D. A.; Strano, M. S.; Nat. Mater. 2005, 4, 86

85. Heller, D. A.; Jeng, E. S.; Yeung, T-K.; Martinez, B. M.; Moll, A. E.; Gastala, J. B.; Strano, M. S.; Science 2006, 311, 511.

86. http://www.fisica.ufc.br/redenano, acessada em Julho 2007 